\title{
Reisestipendien für Kongresse
}

ie Deutsche Gesellschaft für Allergologie und klinische Immunologie (DGAI) möchte die nationale und internationale Präsenz ihrer jungen Mitglieder fördern und stellt wie in der Vergangenheit auch für die Jahre 2000 und 2001 weiterhin Reisestipendien für nationale und internationale Kongresse zur Verfügung. Antragsteller dürfen höchstens 35 Jahre alt sein und von keiner anderen Quelle eine Unterstützung erhalten. Bitte richten Sie Ihre Anfrage an den Vorstand der DGAI (Sekretariat Frau Ratzinger).

Prof. Dr. Claus Bachert

Schatzmeister der DGAI
Prof. Dr. Dr. Johannes Ring

Präsident der DGAI

\section{In memoriam} Dr. med. Helmut Gall

Am 7. 9. 2000 ist Dr. med. Helmut Gall nach kurzer, heftiger Krankheit überraschend für immer von uns gegangen.

Geboren am 4.3.1942 im niederbayrischen Straubing studierte er nach Abitur und Wehrdienst Medizin an der Universität Würzburg und promovierte 1971 magna cum laude in der Anatomie in Würzburg. Im Januar 1976 legte er die Facharztprüfung für Dermatologie ab. Am 1.4.1978 übernahm er am Bundeswehrkrankenhaus Ulm die Leitung der Fachärztlichen Untersuchungsstelle für Dermatologie und Venerologie. 1980 erwarb Gall die Zusatzbezeichnung Allergologie, 1996 die der Umweltmedizin. Im weiteren Verlauf stellte er vor allem in den Arbeitsgebieten Allergologie und Berufsdermatologie sein spezifisches Wissen und seine hohe klinische Kompetenz immer wieder unter Beweis. Es entsprach seinem Selbstverständnis, nicht nur zur Aufklärung, sondern auch zur Weitergabe klinisch relevanter allergologischer Sachverhalte an praktizierende Dermatologen und Allergologen berufen zu sein. Daher verdankt ihm das "Allergo Journal" zahlreiche interessante Fallberichte und Erstbeschreibungen allergischer Reaktionen.

Die Verpflichtung zur Aus- und Fortbildung war für ihn kein Lippenbekenntnis, sondern wurde von ihm in zahllosen Vorträgen und nicht zuletzt im Rahmen der Fora allergologica, die er alljährlich organisierte, praktiziert. Neben seinen vielen klinischen Verpflichtungen betreute er mehr als ein Dutzend Doktoranden sowie zahlreiche klinische Prüfungen und experimentelle Untersuchungen, zuletzt ein Sonderforschungsprojekt über die Prävalenz von Latexallergien.

Pflichterfüllung, Wahrhaftigkeit und Loyalität zu Untergebenen und Kollegen wie zu seinen Vorgesetzten, im Zeitalter der Selbstverwirklichung - oft um scheinbar jeden Preis - keineswegs häufig angewandte Maximen des Handelns im medizinischen, schon gar nicht im universitären Alltag, waren für ihn Selbstverständlichkeiten, die zu praktizieren und Untergebenen, Patienten, Mitarbeitern und Kollegen beispielhaft vorzuleben er bis zuletzt nicht müde wurde. Seine fachliche Kompetenz verlieh ihm die natürliche Autorität, die ohne Gepränge auskommt und auch mit leisen Tönen zur Erfüllung der gemeinsamen Aufgabe zu motivieren vermag. In seiner täglichen Arbeit praktizierte er Kollegialität, ohne durch Standescodices dazu verpflichtet werden zu müssen; mittels der rückhaltlosen Anerkennung der Fachwelt, die ihm über Jahre und Jahrzehnte konstant entgegengebracht wurde, trug er wesentlich zum Ansehen der Dermatologie als Fach und als Institution in Ulm sowohl bei Vertretern anderer klinischer Fächer als auch in der interessierten Öffentlichkeit wie in der veröffentlichten Meinung bei.

Herr Dr. Helmut Gall hinterlässt nicht nur in fachlicher Hinsicht eine schwer zu füllende Lücke. Wir werden ihn nicht vergessen.

RalfU. Peter (Ulm) 